\title{
Genetically Determined Variation in Polyunsaturated Fatty Acid Metabolism May Result in Different Dietary Requirements
}

\author{
Berthold Koletzko a, Hans Demmelmaira, \\ Linda Schaeffer ${ }^{\mathrm{a}, \mathrm{b}}$, Thomas Illig ${ }^{\mathrm{b}}$, Joachim Heinrich ${ }^{\mathrm{b}}$
}

\begin{abstract}
aDivision of Metabolic Diseases and Nutritional Medicine, Dr. von Hauner Children's Hospital, University of Munich, Munich, ${ }^{b}$ Helmholtz Institute of Epidemiology, Neuherberg, Germany
\end{abstract}

\begin{abstract}
Tissue availability of polyunsaturated fatty acids (PUFAs) is of major relevance for health, and it depends on both dietary intake and metabolic turnover. We found close associations between variants in the human genes of $\Delta 5$ - and $\Delta 6$-desaturase, FADS1 and FADS2, and serum phospholipid contents of PUFAs and long-chain PUFAs (LCPUFAs). Polymorphisms and reconstructed haplotypes of FADS1 and the upstream region of FADS2 showed strong associations with levels of the n-6 LC-PUFA arachidonic acid (20:4n-6). Carriers of the less common polymorphisms and their respective haplotypes also had a lower prevalence of allergic rhinitis and atopic eczema. Our data demonstrate for the first time that the fatty acid composition of serum phospholipids is genetically controlled by the FADS1 FADS2 gene cluster. The investigated single nucleotide polymorphisms in this cluster explain $28 \%$ of the variance of serum phospholipid arachidonic acid and up to $12 \%$ of its precursor acids. Based on this genetic variation, individuals may require different amounts of dietary PUFAs or LC-PUFAs to achieve comparable biological effects. We strongly recommend including analyses of FADS1 and FADS2 polymorphism in future cohort and intervention studies addressing the biological effects of PUFAs and LC-PUFAs, which should enhance the sensitivity and precision of such studies.
\end{abstract}

The metabolic availability of polyunsaturated fatty acid acids (PUFAs) has a major impact on human health. PUFA status has been related, among other outcomes, to early visual, cognitive and motor development [1, 2], mental 


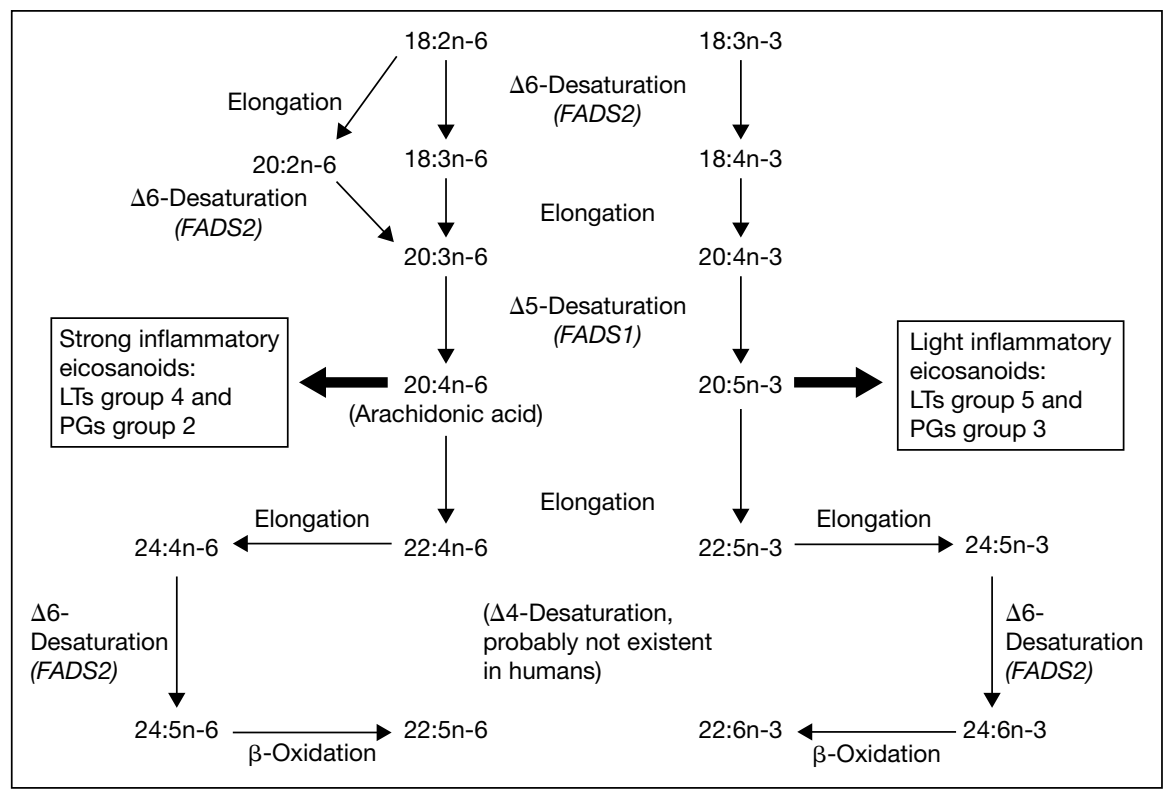

Fig. 1. Pathways for the conversion of $n-6$ (left) and n-3 (right) essential fatty acids into LC-PUFAs by enzymatic desaturation and chain elongation. Modified from Schaeffer et al. [15].

health and psychiatric disorders [3], cardiovascular disease mortality [4], immunological and inflammatory responses as well as related diseases such as allergies [5, 6]. These and other biological effects of PUFAs appear to be mediated to a large part by the availability of the long-chain PUFAs (LCPUFAs) with $\geq 20$ carbon atoms and $\geq 3$ double bonds, such as the n- 6 LCPUFA arachidonic acid (20:4n-6), and the n-3 LC-PUFAs eicosapentaenoic acid (20:5n-3) and docosahexaenoic acid (22:6n-3; fig. 1). Preformed LCPUFAs are also supplied with some foods (e.g. arachidonic acid with meats and eggs; eicosapentaenoic acid and docosahexaenoic acid with marine foods). Dietary LC-PUFA supply has a marked effect on blood and tissue contents which has been documented in numerous studies [7]. LC-PUFAs can also be derived in human metabolism from the precursor essential fatty acids, linoleic acid (18:2n-6) and $\alpha$-linolenic acid (18:3n-3), by consecutive desaturation and chain elongation (fig. 1 ).

The activity of PUFA desaturation and chain elongation appears to be very limited in humans [8], but there are numerous indications for considerable inter-individual variation in the capacity for endogenous formation of LCPUFAs. For example, in a study published almost 2 decades ago, we found a 


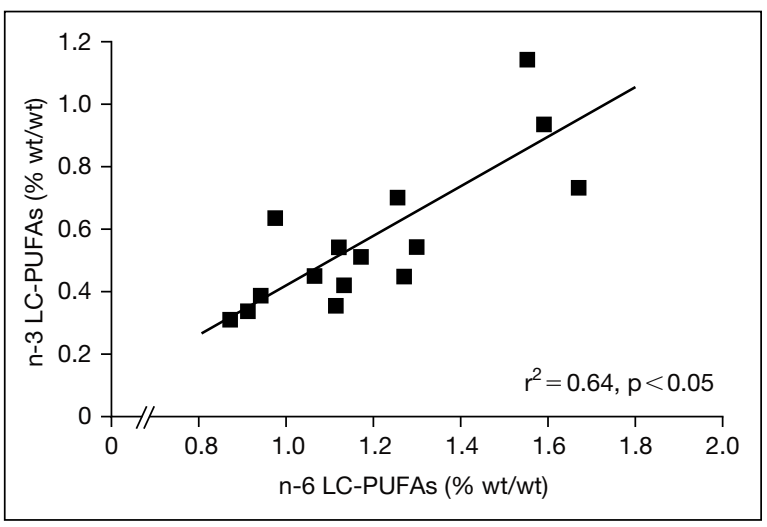

Fig. 2. Correlation of n-3 and n-6 LC-PUFA contents in mature human milk, suggesting inter-individual differences in the ability to form LC-PUFAs. Modified from Koletzko et al. [9].

rather close correlation of n-6 and n-3 LC-PUFA contents in mature human milk [9] (fig. 2), even though the main dietary sources of the two LC-PUFA families are very different. Thus, it appears that some women have a higher ability to synthesize and secrete milk LC-PUFAs of both the n- 6 and the n-3 series than others.

A similar conclusion was drawn by Innis et al. [10] who observed a positive correlation of docosahexaenoic acid and n- 6 docosapentaenoic acid in red blood cell phosphoglycerides of preschool children, in contrast to the reciprocal relationship found in animals where high n-6 docosapentaenoic acid levels are a marker of low docosahexaenoic acid availability. The authors concluded that the positive relationship of n-6 and n-3 LC-PUFA levels observed in these children may reflect different activity levels of desaturases between individuals affecting the conversion of both the n- 6 and the n- 3 fatty acids.

Recently we repeatedly studied the dietary intake and fatty acid composition of plasma phospholipids in a group of healthy children at the ages of 24, 36 and 60 months. As one might expect, dietary fatty acid intake patterns changed markedly over time from the age of 2 to 5 years, and there was no correlation or 'tracking' of dietary intakes of saturated, monounsaturated and polyunsaturated fatty acid over time. In contrast, there was a significant correlation ('tracking') over the 3 time points of plasma phospholipid levels of n-6 LC-PUFA $(r=0.67)$ and the arachidonic acid/linoleic acid ratio $(\mathrm{r}=0.64)$, and to a lesser degree also of n-3 LC-PUFA $(r=0.32)$ and of the docosahexaenoic acid/ $\alpha$-linolenic acid ratio $(r=0.32)$ [11]. This tracking of plasma LC-PUFA levels in the absence of tracking of dietary intake patterns leads us to conclude that there is inter-individual variation in the ability to endogenously synthesize LC-PUFAs among 
these children, which persists over time and could most likely be due to genetically determined differences in metabolic turnover. Considerable inter-individual differences in endogenous PUFA conversion have also been demonstrated in stable isotope studies $[12,13]$, but these data do not reveal to which extent such differences in PUFA conversion may be due to moderating nutritional, metabolic and endocrine factors [8], or due to genetic variation.

The enzymes $\Delta 5$ - and $\Delta 6$-desaturase are considered rate-limiting for the enzyme-mediated conversion steps of this pathway [8]. Both desaturases are expressed in the majority of human tissues, with the highest levels in liver but considerable activity also found in the brain, heart, and lung. The hypothesis that they play a key role in inflammatory diseases is strengthened by functional studies in mice, in which selective $\Delta 5$ - and $\Delta 6$-desaturase inhibitors induced marked anti-inflammatory effects [14].

In human tissue, the regulatory mechanisms of $\Delta 5$ - and $\Delta 6$-desaturases have scarcely been examined. The human desaturase cDNAs were first cloned in 1999 and identified in 2000 as fatty acid desaturase-1 (FADS1, encoding $\triangle 5$ desaturase) and fatty acid desaturase-2 (FADS2, encoding $\Delta 6$-desaturase) in the human genome [15]. The two genes are located in a cluster on chromosome 11 (11q12-13.1) with a head-to-head orientation. The sequence of the FADS1 FADS2 gene cluster can be identified as a region of conserved synteny to the mouse genes Fads1 and Fads2 coding for the homologous enzymes on mouse chromosome 18, with the same head-to-head orientation and the same homologous adjacent genes (http://www.ensembl.org/Homo_sapiens/syntenyview). Linkage was previously reported between or nearby the human chromosomal region 11q12-13.1 and complex diseases like type-1 diabetes, osteoarthritis, and bipolar disorders, as well as asthma, atopy and allergy-related quantitative traits such as total and specific IgE levels [15].

\section{Evaluation of the Effects of FADS1 and FADS2 Polymorphisms on LC-PUFA Status in Humans}

In an attempt to explore the genetic determinants of PUFA metabolism, we performed an analysis of 18 single nucleotide polymorphisms (SNPs) of the FADS1 FADS2 gene cluster in 727 adult volunteers participating in the European Community Respiratory Health Survey I (ECRHS I) [15]. In 1991-1992 a population-based sample of randomly selected, mainly Caucasian subjects aged between 20 and 64 years, was recruited in the city of Erfurt, Germany. Of the cohort of 1,282 participants who answered the main questionnaire, samples from 727 participants were available for genetic testing of DNA, and deep frozen $\left(-80^{\circ} \mathrm{C}\right)$ serum samples were used for analysis of phospholipid fatty acid contents. The study protocol was approved by the Ethics Committee of the Bavarian Board of Physicians at Munich. The analyzed SNPs were chosen from data reported in public databases on the basis of 
positional and functional aspects to enhance the chance of detecting associations [15]. Genotyping of SNPs in the FADS1 FADS2 cluster was performed using matrix-assisted laser desorption/ionization time-of-flight mass spectrometry (MALDI-TOF MS) to detect allele-specific primer extension products (Mass Array, Sequenom, San Diego, Calif., USA).

The results of genotyping confirmed that 18 SNPs reported in public databases in the FADS1 FADS2 cluster were polymorphic, with a mean genotyping success rate of $97.4 \%$. The distributions of genotypes for all analyzed SNPs were consistent with Hardy-Weinberg equilibrium. Redundant markers (rs174545, rs174546, and rs174568) were left out of the analyses. Association analysis of SNPs with fatty acids showed highly significant results for the majority of the SNPs in the FADS1 FADS2 cluster and the n-6 and n-3 fatty acids ( $p$ values $<1.0^{*} 10^{-13}$ ) except for $n-6$ docosapentaenoic acid (22:5n-6) and docosahexaenoic acid (22:6n-3). Subjects carrying the minor alleles of the SNPs rs174544, rs174553, rs174556, rs174561, rs174568, rs968567, rs99780, rs174570, rs2072114, rs174583, and rs174589 exhibited enhanced levels of the precursor fatty acids linoleic acid (18:2n-6), eicosadienoic acid (20:2n-6), dihomo- $\gamma$-linolenic acid (20:3n-6), and $\alpha$-linolenic acid (18:3n-3), and decreased levels of the product fatty acids $\gamma$-linolenic acid (18:3n-6), arachidonic acid (20:4n-6; fig. 3), adrenic acid (22:4n-6), eicosapentaenoic acid (20:5n-3), and n-3 docosapentaenoic acid (22:5n-3) [15].

Haplotypes were statistically reconstructed for two different windows (table 1). To avoid large reconstruction errors resulting from missing data, the reconstruction was based only on persons from whom all genotypes were available. The strongest associations were found in the first part of the gene cluster. The second window was restricted to the first five strongly correlated SNPs with $\mathrm{r}^{2}>0.7$, for which complete genotype data of 637 subjects was available. For the 5-locus haplotypes, only two haplotypes had a frequency $>5 \%$, with the most common haplotype carrying the major alleles at all loci (frequency 68\%), and with the next frequent haplotype carrying only minor alleles (frequency 26\%). Haplotype association analysis indicated highly significant associations also after correction for multiple testing ( $p$ values multiplied by 17 for the 17 tested outcomes) between the haplotypes and the fatty acid levels (examples for linoleic and arachidonic acid are shown in table 2). Virtually all haplotypes carrying minor alleles were associated $\left(\mathrm{p}<1.0^{*} 10^{-13}\right)$ with increased levels of linoleic acid (18:2n-6), eicosadienoic acid (20:2n-6), dihomo- $\gamma$-linolenic acid (20:3n-6), and $\alpha$-linolenic acid (18:3n-3), and with decreased levels of $\gamma$-linolenic acid (18:3n-6), arachidonic acid (20:4n-6), adrenic acid (22:4n-6), eicosapentaenoic acid (20:5n-3), and n-3 docosapentaenoic acid (22:5n-3), which was in line with the findings of the SNP analysis. For n-6 docosapentaenoic acid (22:5n-6, after correction for multiple testing) and docosahexaenoic acid (22:6n-3, uncorrected and corrected), haplotype analysis - as the previous SNP association analysis - showed no significant result, presumably because the effect of desaturation activity on 


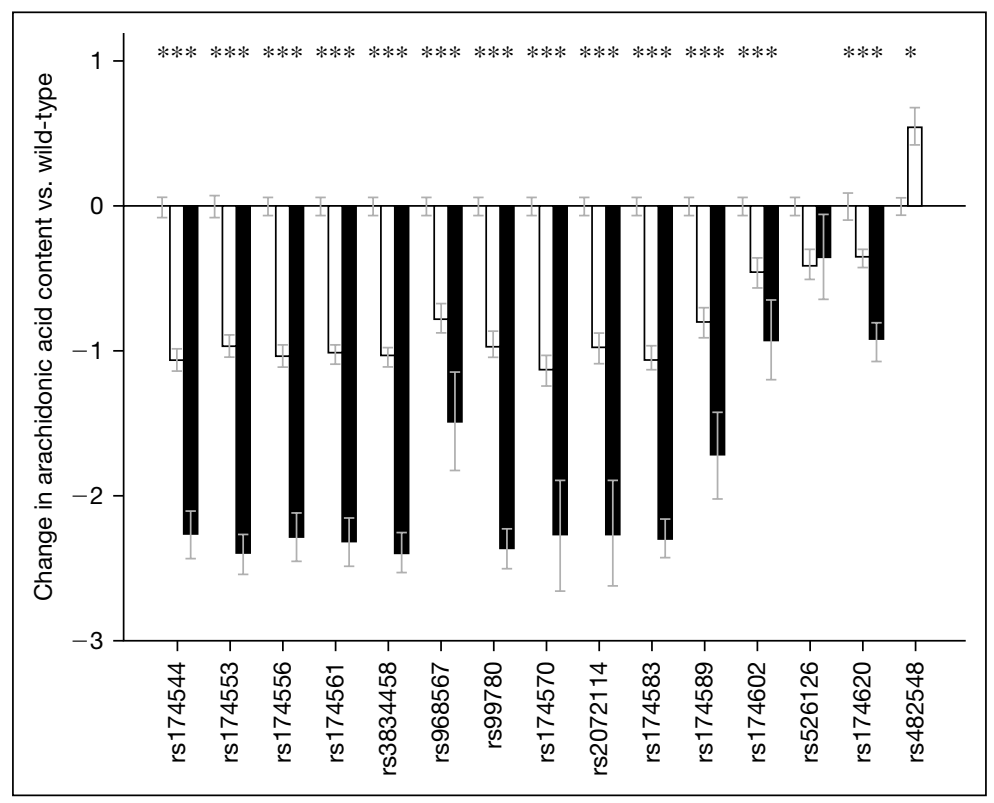

Fig. 3. Association analysis of 15 SNPs with changes in the serum phospholipid contents of the major n-6 LC-PUFA arachidonic acid (20:4n-6) versus mean values for subjects carrying the respective major SNP on both alleles [major A major A]. $\square=$ Changes in fatty acid composition for heterozygous carriers of the minor SNP (level [major A, minor a] minus level [major A, major A]); $\mathbf{\square}=$ homozygotes of the minor allele (level [minor a, minor a] minus level [major A, major A]). Heterozygotes for the minor allele of the SNP rs 174544 have a lower level of arachidonic acid (9.2\%) in comparison to homozygotes for the major allele (10.2\%), whereas those homozygous for that minor allele exhibit only $8 \%$ arachidonic acid (means $\pm \mathrm{SE}$ ). $* \mathrm{p}<0.05 ; * * * \mathrm{p}<0.01$. Adapted from Schaeffer et al. [15].

their plasma concentration is moderated by the indirect synthesis via peroxisomal $\beta$-oxidation.

Genotypes or haplotypes were not associated with total or specific IgE levels, but carriers of the minor alleles of several SNPs had significantly reduced odds ratios (ORs) for allergic rhinitis and atopic eczema: allergic rhinitis, rs174544 $(\mathrm{OR}=0.59,95 \%$ CI 0.36, 0.99), and rs2072114 (OR $=0.45,95 \%$ CI 0.23, 0.88), and atopic eczema, rs174556 (OR $=0.49,95 \%$ CI 0.25, 0.94). The 5-locus haplotype consisting only of minor alleles showed protective ORs with allergic rhinitis (OR $=0.46,95 \%$ CI $0.26,0.83)$ as well as with atopic eczema (OR $=0.46$, 95\% CI $0.22,0.94)$. The 11-locus haplotypes also showed a tendency towards protective ORs. Thus, in this population-based sample we found that minor alleles of SNPs in the FADS1 gene and the promoter region of the FADS2 gene, the 
Table 1. Haplotype characteristics for 11-locus and 5-locus haplotypes

\begin{tabular}{|c|c|c|c|}
\hline Haplotype & & Alleles $^{\mathrm{a}}$ & Frequency, \% \\
\hline \multicolumn{4}{|c|}{ 11-locus haplotype (rs174544-rs174589) } \\
\hline $\mathrm{MaA}^{\mathrm{b}}$ & CACTTGCCACG & 11111111111 & 68.8 \\
\hline Haplo.1 & AGTCdelATCATC & 22222221122 & 10.6 \\
\hline Haplo.2 & AGTCdelGTTGTG & 22222122221 & 7.8 \\
\hline Haplo.3 & CGCTdelGTTATC & 12112122122 & 2.9 \\
\hline Haplo.4 & AGTCdelGTCGTG & 22222121221 & 2.6 \\
\hline Haplo.5 & AGTCdelATCGTG & 22222221221 & 1.2 \\
\hline Haplo. 6 & AGTCdelATCATG & 22222221121 & 1.0 \\
\hline $\begin{array}{l}\text { Haplo.rare } \\
\text { (frequency <1\%) }\end{array}$ & & & 5.0 \\
\hline \multicolumn{4}{|c|}{ 5-locus haplotype (rs17544-rs3834458) } \\
\hline $\mathrm{MaA}$ & CACTT & 11111 & 68.6 \\
\hline $\mathrm{MiA}^{\mathrm{c}}$ & AGTCdel & 22222 & 25.7 \\
\hline Haplo.1 & CGCTdel & 12112 & 3.5 \\
\hline $\begin{array}{l}\text { Haplo.rare } \\
\text { (frequency }<1 \% \text { ) }\end{array}$ & & & 2.3 \\
\hline $\begin{array}{l}\text { Adapted from Sc } \\
{ }^{\mathrm{a}} 1=\text { Major allele } \\
{ }^{\mathrm{b}} \mathrm{MaA}=\text { Major al } \\
{ }^{\mathrm{c}} \mathrm{MiA}=\text { Minor al }\end{array}$ & $\begin{array}{l}\text { er et al. [15]. } \\
\text { minor allele. } \\
\text { taplotype carrying o } \\
\text { laplotype carrying on }\end{array}$ & $\begin{array}{l}\text { mmon alleles. } \\
\text { re allele. }\end{array}$ & \\
\hline
\end{tabular}

two genes encoding the two relevant desaturases on the metabolic pathway leading to arachidonic acid (20:4n-6) production, as well as their corresponding haplotypes were highly associated with an increase in levels of linoleic acid (18:2n-6), eicosadienoic acid (20:2n-6), dihomo- $\gamma$-linolenic acid (20:3n-6), and $\alpha$-linolenic acid (18:3n-3), and with a decrease in the levels of $\gamma$-linolenic acid (18:3n-6), arachidonic acid (20:4n-6), adrenic acid (22:4n-6), eicosapentaenoic acid (20:5n-3), and n-3 docosapentaenoic acid (22:5n-3), and less pronounced in n-6 docosapentaenoic acid (22:5n-6) in human serum phospholipids. The most significant associations and the highest proportion of genetically explained variability (28\%) were found for arachidonic acid (20:4n-6; table 3).

\section{Implications of the Observed Genetic Effects on PUFA and LC-PUFA Status in Humans}

Our findings highlight the contribution of the desaturation pathways on n-6 and n-3 PUFA and LC-PUFA levels in serum phospholipids, and the major importance of its genetic control. Not only arachidonic acid (20:4n-6), but also 
Table 2. Examples of associations of FADS1 FADS2 haplotypes with the major dietary essential fatty acid, linoleic acid (18:2n-6), and the major n-6 LC-PUFA, arachidonic acid (20:4n-6)

\begin{tabular}{llcc}
\hline Haplotype & & C18:2n-6 & C20:4n-6 \\
\hline Haplo.1 & $\mathrm{p}$ & $8.5^{*} 10^{-6}$ & $3.7^{*} 10^{-15}$ \\
& Coefficient & 1.18 & -1.03 \\
Haplo.2 & $\mathrm{p}$ & 0.0066 & $<1.0^{*} 10^{-14}$ \\
& Coefficient & 0.95 & -1.48 \\
Haplo.3 & $\mathrm{p}$ & 0.0060 & $3.2^{*} 10^{-7}$ \\
& Coefficient & 1.518 & -1.266 \\
Haplo.4 & $\mathrm{p}$ & 0.086 & $9.4^{*} 10^{-5}$ \\
& Coefficient & 1.311 & -1.124 \\
Haplo.5 & $\mathrm{p}$ & 1.0 & 0.045 \\
& Coefficient & -0.29 & -1.066 \\
Haplo.6 & $\mathrm{p}$ & 0.16 & 0.22 \\
& Coefficient & 1.907 & -0.955 \\
Haplo.rare & $\mathrm{p}$ & 0.079 & 0.068 \\
& Coefficient & 1.067 & -0.564 \\
MiA-Haplotype & $\mathrm{p}$ & $1.2 * 10^{-13}$ & $<1.0^{*} 10^{-14}$ \\
& Coefficient & 1.18 & -1.17 \\
Haplo.1 & $\mathrm{p}$ & 0.024 & $1.3 * 10^{-7}$ \\
& Coefficient & 1.177 & -1.122 \\
Haplo.rare & $\mathrm{p}$ & 0.32 & 0.70 \\
& Coefficient & 1.067 & -0.482 \\
\hline p values and $\beta$-coefficients (change of fatty acid or change of log of fatty acid per \\
copy of the haplotype) are given. p values that exceed 1.0 after correction for multiple \\
testing have been marked down to 1.0. Adapted from Schaeffer et al. [15]. \\
\hline
\end{tabular}

Table 3. Percentage of variations in the fatty acid levels explained by the SNPs for the models containing the SNPs of the 5-locus and 11-locus haplotypes

\begin{tabular}{lcc}
\hline Fatty acid & $\mathrm{R}^{2} 5$ SNPs $\%$ & $\mathrm{R}^{2} 11$ SNPs \% \\
\hline Linoleic acid (C18:2n-6) & 8.6 & 9.2 \\
$\gamma$-Linolenic acid (ln C18:3n-6) & 7.9 & 7.9 \\
Eicosadienoic acid (C20:2n-6) & 10.1 & 12.3 \\
Dihomo- $\gamma$-linolenic acid (C20:3n-6) & 7.4 & 10.8 \\
Arachidonic acid (C20:4n-6) & 27.7 & 28.5 \\
Adrenic acid (C22:4n-6) & 6.3 & 5.9 \\
n-6 Docosapentaenoic acid (ln C22:5n-6) & 1.2 & 1.5 \\
$\alpha$-Linolenic acid (C18:3n-3) & 3.9 & 6.4 \\
Eicosapentaenoic acid (ln C20:5n-3) & 5.2 & 5.1 \\
n-3 Docosapentaenoic acid (C22:5n-3) & 5.5 & 2.9 \\
Docosahexaenoic acid (C22:6n-3) & 1.4 & \\
\hline
\end{tabular}


its precursors, linoleic acid (18:2n-6), $\gamma$-linolenic acid (18:3n-6) and dihomo$\gamma$-linolenic acid (20:3n-6), showed strong associations with the genetic variants. In free-living individuals with self-selected diets, the reconstructed haplotypes explain a major proportion of the variation in plasma phospholipid content, particularly of arachidonic acid where about $28 \%$ of variation of blood levels is due to genetic variation (table 3), whereas the value is in the order of $10 \%$ for the precursor fatty acids of arachidonic acid. Smaller percentage values are found for n-3 fatty acids, which may reflect a higher importance and a larger degree of variation in dietary intakes of both the precursor $\alpha$-linolenic acid primarily from vegetable oils and the products eicosapentaenoic acid and docosahexaenoic acid primarily from marine foods.

\section{Conclusion}

(1) Blood lipid levels of both PUFAs with 18 carbon atoms that are conventionally referred to as the essential fatty acids, and of their biologically active LC-PUFA derivatives, are influenced not only by diet, but to a large degree also by genetic variants commonly found in a European population.

(2) Levels of docosahexaenoic acid are not significantly affected by the SNPs studied, but dietary supply is the primary determinant of docosahexaenoic acid values.

(3) The studied SNPs for fatty acid desaturation enzymes are associated with allergic rhinitis and atopic dermatitis which supports the conclusion that the availability of PUFAs affects allergic endpoints.

(4) At the same level of dietary intake of precursor PUFAs or LC-PUFAs, the respective biological and health effects may markedly differ due to genetic differences in their metabolism.

(5) Based on genetic variation, subgroups of the population may have different requirements of dietary PUFA or LC-PUFA intakes, respectively, to achieve comparable biological effects.

(6) These relationships should be explored in more detail in different populations.

(7) We strongly recommend including analyses of FADS1 and FADS2 polymorphism in future cohort and intervention studies addressing the biological effects of PUFAs and LC-PUFAs, which should also lead to enhanced sensitivity and precision of such studies.

\section{Acknowledgements}

This work was partly funded by German Research Foundation (Deutsche Forschungsgemeinschaft, Bonn, Germany), research grants HEI 3294/1-1 and KO 912/8-1, BMBF (NGFN), the SFB-386 (DFG), and by a travel grant from the 
Boehringer Ingelheim Fonds. B.K. is the recipient of a Freedom to Discover Award of the Bristol Myers Squib Foundation, New York, N.Y., USA.

\section{References}

$\checkmark 1$ Beblo S, Reinhardt H, Demmelmair H, et al: Effect of fish oil supplementation on fatty acid status, coordination and fine motor skills in children with phenylketonuria. J Pediatr 2007; 150:479-484.

-2 Koletzko B, Cetin I, Brenna JT; the Perinatal Lipid Intake Working Group: Dietary fat intakes for pregnant and lactating women. Br J Nutr 2007;98:873-877.

-3 Muskiet FA, Kemperman RF: Folate and long-chain polyunsaturated fatty acids in psychiatric disease. J Nutr Biochem 2006;17:717-727.

4 Leaf A: Prevention of sudden cardiac death by n-3 polyunsaturated fatty acids. Fundam Clin Pharmacol 2006;20:525-538.

5 Kompauer I, Demmelmair H, Koletzko B, et al: Association of fatty acids in serum phospholipids with hay fever, specific and total immunoglobulin E. Br J Nutr 2005;93:529-535.

6 Trak-Fellermeier MA, Brasche S, Winkler G, et al: Food and fatty acid intake and atopic disease in adults. Eur Respir J 2004;23:575-582.

7 Krauss-Etschmann S, Shadid R, Campoy C, et al: Effects of fish-oil and folate supplementation of pregnant women on maternal and fetal plasma concentrations of docosahexaenoic acid and eicosapentaenoic acid: a European randomized multicenter trial. Am J Clin Nutr 2007;85:1392-1400.

8 Krohn K, Demmelmair H, Koletzko B: Macronutrient requirements for growth: fat and fatty acids; in Duggan C, Watkins JB, Walker WA (eds): Nutrition in Pediatrics. Basic Science and Clinical Applications, ed 4. Hamilton, Decker, 2008, chapt 7.

9 Koletzko B, Mrotzek M, Bremer HJ: Fatty acid composition of mature human milk in Germany. Am J Clin Nutr 1988;47:954-959.

10 Innis SM, Vaghri Z, King DJ: n-6 Docosapentaenoic acid is not a predictor of low docosahexaenoic acid status in Canadian preschool children. Am J Clin Nutr 2004;80:768-773.

11 Guerra A, Demmelmair H, Toschke AM, Koletzko B: Three-year tracking of fatty acid composition of plasma phospholipids in healthy children. Ann Nutr Metab 2007;51:433-438.

12 Del Prado M, Villalpando S, Elizando A, et al: Contribution of dietary and newly formed arachidonic acid to human milk lipids in women eating a low fat diet. Am J Clin Nutr 2001;74: $242-247$.

13 Demmelmair H, von Schenck U, Behrendt E, et al: Estimation of arachidonic acid synthesis in full term neonates using natural variation of $13 \mathrm{C}$ content. J Pediatr Gastroenterol Nutr 1995;21:31-36.

14 Obukowicz MG, Welsch DJ, Salsgiver WJ, et al: Novel, selective delta6 or delta5 fatty acid desaturase inhibitors as antiinflammatory agents in mice. J Pharmacol Exp Ther 1998;287: $157-166$.

15 Schaeffer L, Gohlke H, Müller M, et al: Common genetic variants of the FADS1 FADS2 gene cluster and their reconstructed haplotypes are associated with the fatty acid composition in phospholipids. Hum Mol Genet 2006;15:1745-1756.

\section{Discussion}

Dr. Isolauri: Thank you very much for these new aspects and challenges to the food industry. Concerning the data on the effect of n- 6 fatty acids and margarine in allergy: are all margarines the same in $n-6$ composition and how well does spread on bread reflect the whole fatty acid intake?

Dr. Koletzko: Margarine is only one of many factors affecting n-6 intake. Margarines differ quite a bit in their composition. There are more and more margarines based on rape seed oil which has less linoleic acid and more oleic acid. There 
is quite a variation but these were epidemiological studies which just looked at associations between dietary patterns and outcomes, and there are a number of studies with large sample sizes that found an association which might or might not be due to linoleic acid intake. We actually looked at this in an adult population, in a school-age population, and in birth cohorts. In the adult population we found that both the dietary intake of arachidonic acid and the plasma levels of arachidonic acid are to some extent associated with allergic end points, allergic sensitization and allergic disease. The data are not very clear cut and there are somewhat different results in males and females which might be explained by different dietary patterns and different reporting patterns between males and females, but there is some association. If there is such a strong effect of genetic variation, dietary data alone will not give the full picture.

Dr. Prescott: Thank you very much for your very important work which clearly illustrates that we need to look at this in our studies. In our work in Perth we have recently shown that fatty acid supplementation in pregnancy can modify T-cell signaling pathways, particularly protein kinase $\mathrm{C}$ isozymes which we also showed had a protective effect on allergic disease. Clearly we need to talk about looking at potential polymorphisms and how that might modulate these effects. We also have another larger cohort of 400 children in which we are looking at the effect of fish oil supplementation on early immune function and allergy prevention. My question is how do you think that high dose fish oil in pregnancy may have epigenetic effects on the baby?

Dr. Koletzko: Those are obviously issues that one might want to study and you clearly have populations to look at that. If I recall correctly your first study was in 100 mothers who had a very high dose of long-chain $n-3$, which is a huge dose relative to the potential impact on endogenous metabolism. We have done a similar study with 300 women using a lower dose of $500 \mathrm{mg}$ DHA and the results are very similar to yours in terms of modification of immune phenotypes in the newborn infants. I am very excited about the potential of using omega-3 fatty acids as a preventive intervention in pregnancy. Perhaps we should join our cohorts together to search for polymorphisms. We have material left that could be used for epigenetic analysis and I think this would be an exciting opportunity.

Dr. Waterland: You spoke of these different haplotypes consisting of clusters of SNPs, but where were the SNPs located? Were they within the coding regions or within the regulatory regions of the genes? The contra-oriented juxtaposition position of FADS1 and FADS2 suggests the potential for a shared regulatory region. I wonder if you have explored the potential epigenetic regulation of those two genes?

Dr. Koletzko: We certainly did not look at epigenetic aspects in this study. We just had DNA available from these subjects and we looked at the polymorphisms. The SNPs were primarily exon SNPs with functional relevance although exon SNPs without known functional relevance were not fully excluded.

Dr. Hofstratt: Is there a kind of market for having a genetic test go with a margarine or other food stuffs?

Dr. Koletzko: That is the whole question behind personalized nutrition, where we come to a situation in which people choose their food products and make dietary choices according to differences in biological predisposition and genetic variation. At least in Europe I am not sure that in the near future people will go into a supermarket and take a quick blood test and then look for the products that are labeled green, blue and red. I am not sure this is within reach for the general consumer market, but it is certainly in reach for clinical nutrition. For example fish oil interventions are now used in intensive care with the concept that an inflammatory response can be reduced and outcome improved. In a clinical setting I can see that happening with the rapidly advancing methodology. With pharmacological agents we test for genetic polymorphisms 
before we give a drug we could do the same with the fatty acid polymorphisms to dose the fish oil intervention. Will this be happening with respect to the general consumer market? What would consumer research at Nestlé say? Is this something that we can expect in the next 15 or 20 years?

Dr. Rinaldo: I work in a biochemical genetics laboratory and in recent years we have faced a dramatic increase in requests to measure polyunsaturated fatty acids (PUFAs) for all sorts of studies beyond my primary area of interest which is inherited disorders of fatty acid oxidation. We are testing patients who are under lipid-restricted diets. A major issue that came up in these studies was that historically plasma PUFA results have been expressed as a percent of the total amount. Once I showed our collaborators and colleagues results expressed using absolute amounts, and some of the variabilities in plasma you mentioned earlier disappeared. In your presentation, your results were expressed as a percent of a total, so I would like to know if the reported differences between genders and other variabilities could be a confounding factor in the evaluation of this polymorphism?

Dr. Koletzko: A number of people have looked at that comparing absolute concentrations, milligrams per liter for example, to percentage of fatty acids in e.g. phospholipids or total lipids. It is a complex question but my understanding of the literature and also my experience with our own measurements is that the percentage data show much less variation than the absolute data. If there is a change in the total concentration of lipid fractions, if the lipoproteins go up and down, the relative proportion of fatty acids does not necessarily change. Moreover, there are a number of studies which have associated end points with plasma levels, and it was clearly shown that the correlation to phospholipid percentage data was much closer than to the absolute data, and also much closer than to the red blood cell levels, which are often considered the best determinant of fatty acid status but I have considerable doubts. With respect to the inborn errors I agree with you that there are a number of unresolved questions. We also looked at samples from $\beta$-oxidation disorder patients and we have often found extremely low DHA values and patterns with classical essential fatty acid deficiency with an increased Mead acid, which is clearly a problem that needs to be looked at in further detail. We have indications from a population of children with phenylketonuria (PKU) that the supply matters. Both our group [1] and the group in Milan [2] have shown that DHA supplementation to children with PKU improves electrophysiology, latency time, and speed of information processing. In the Journal of Pediatrics we also published that supplementation with DHA in PKU children improves fine motor coordination and some other simpler behavioral responses [3]. Yes, there is really room to look at this in further detail in children on restricted diets.

Dr. Rinaldo: That is exactly the point; in some patients with long-chain fatty acid oxidation disorders the percent estimate indicated a DHA deficiency when the values were looked at as absolute amounts, where actually comfortably within what we had defined as the age-matched reference range, which was really the type of discrepancy I was referring to. What would be your advise; should we overrule a normal absolute amount because as a percent it appears to be low?

Dr. Koletzko: I am not claiming that I have the final answer and we probably need to look at this in further detail, particularly under extreme conditions such as a severely restricted diet which might be very different from other situations. However, the data that are available indicate that percentage values are more predictive of functional response, and in a way it is plausible because if you think of circulating plasma lipids they would be exchanged presumably as a lipid with tissue and not selectively as one fatty acid. This is somewhat speculative and I really suggest we need to look at this further. In the animal world, however, there are also data relating tissue composition much closer to percentage values in plasma. 
Dr. Haschke: I would like to comment on what consumer researchers at Nestlé should do in the future. This is not a subject for consumer research. The matter is much too complex and complicated. If you look at the pharma industry where polymorphisms are clearly associated with different drug metabolisms, the message is not even understood by most medical doctors. There are a few who are focused and apply the methods. As we have learned from the pharma industry, the future for the nutrition industry will be to develop research organizations like the pharma diagnostic sections, which are the most rapidly developing sections in the pharma industry. If you look at Roche or Bayer, for example, their diagnostic sections are just booming. The message must be focused, and the outcome must be affordable because if nobody can pay for the findings, it will be very difficult to transfer them to a consumer message. The prices for chips have come down, one thousand-fold within half a year now. If there is a future for this kind of research, then the nutrition companies are well advised to establish diagnostic sections and also focus on the product outcome just as the pharma industry is doing.

Dr. Koletzko: As you alluded to, the price barrier will no longer be a barrier for diagnostic testing in the foreseeable future, and it will depend on consumers who understand that there is a clear advantage to applying this. If, for example, the results from this first study on the predictive effects on allergic end points are confirmed, then I am sure many families will want to know this and will probably adapt the dietary pattern for their children if there is evidence that this is of benefit for them. In a way we are not far away from that. It is very clear that for a child with inborn errors the dietary pattern can be adapted by focusing on the phenotype or genotype. Many people will also do this if they have a high cholesterol value; they will take dietary advice based on their high cholesterol value and will buy a margarine with phytostanols based on that finding. So I don't see that this is very far away. If people find the information that this is of benefit for them, then I am sure it will happen.

Dr. Gluckman: In our rodent studies we find that FADS1 and FADS2 are two of the most sensitive genes, a life-long expression from fetal undernutrition. So there are probably genes that are already subject to quite a lot of direct or indirect epigenetic regulation. They tend to move together.

Dr. Koletzko: That is exciting information that I would like to hear more about. The question then also rises can we study that in humans and can we find similar patterns?

Dr. Gluckman: The dilemma is what tissue, because this is a matter of the metabolic reactive tissues. I would doubt that you see it just by studying blood. I think that is the dilemma of epigenetics; that it is so often tissue-specific effects as opposed to global effects.

Dr. Koletzko: We assume that most of the desaturation in humans occurs in the liver, and liver biopsies are actually done in humans. If you don't need much material perhaps there are opportunities.

Dr. Laitinen: You started your talk by showing the scheme on n-3 and n-6 fatty acids and their immunological effects, and you also presented the thought that n-3 fatty acids are the good ones: they have a lot of beneficial effects in immunological terms, and this has indeed led to supplementation studies with interesting results as well. But there are also some studies showing that there might be some risks, for example one study looking at n-3 fatty acids in breast milk showed an increased risk of atopy. So what are your thoughts on this increased trend of supplementing with n-3 fatty acids and could these new results of yours on genetic variation contribute to this?

Dr. Koletzko: Obviously we have very little information so far on the effects of the supplementation in the perinatal period in pregnancy and in the lactation period on 
allergic end points. The studies that Dr. Prescott and our group have done do not give sufficient evidence yet to really draw firm conclusions, and we need to look at this further. However, the early nutrition programming project together with the perinatal lipid nutrition project and seven international scientific societies have just brought out a position paper recommending that pregnant and breastfeeding women should aim at achieving a dietary DHA intake averaging at least $200 \mathrm{mg}$ /day based on reduction of early preterm birth, and also based on the reported benefits on outcomes in child development [4]. This is already a very strong data set which this group of 50 international scientists looked at in an evidence-based way, supporting that some supply of long-chain n-3 during pregnancy is beneficial, not necessarily for allergy though, and there we need more good studies.

Dr. Isolauri: At the enterocyte level the effect of $n-6$ fatty acids appears to be suppression of T-cell proliferation and an increase in IL-10 production, and that of n-3 is also associated with dendritic cells producing IgA. Therefore we do not have enough data to support one over the other.

Dr. Koletzko: Everybody in science knows that the world is usually not as simple that there is just good and evil. In the real world, particularly in biology, things are usually more complex and to say arachidonic acid is bad and eicosapentanoic acid is good is probably an over-simplification. It doesn't reflect what is really happening in biology.

Dr. German: Actually now this population has been identified, wouldn't it be a very valuable resource to discover what else is affected by arachidonic acid and therefore likely be beneficially sensitive to things like n-3? You talked about allergic rhinitis; what else do they get?

Dr. Koletzko: This is a cohort that was recruited in 1991 and 1992, and it was recruited with this specific perspective to look at respiratory health and respiratory allergy. So the phenotyping in that area is very good and that is why we took that population because it was convenient: the blood was there, DNA was there, the phenotyping was there, but we don't have a very good description of other outcomes in this population. However, there are other cohorts, we are now working on a cohort of children trying to look at the same question and with different phenotype descriptions. Again I would like to reiterate if any of you is embarking on either cohort or intervention studies grab the opportunity and look at those polymorphisms. If you can't do them yourself we will be happy to collaborate and do it because it is a very simple, high throughput measurement. It would be worthwhile to look at in many different populations and see what the effects might be.

Dr. Gluckman: Are there any gender-specific effects in these studies? I mention this because quite gender-specific effects of other polymorphisms, such as PPAR $\alpha$ polymorphisms, and the relationship between PUFA intake and, e.g., plasma cholesterol or other lipoprotein-related levels in blood have been seen.

Dr. Koletzko: We can't really draw conclusive evidence from the our population. But in the epidemiological studies by Trak-Fellermeier et al. [5] for example, there are differences in the relationship between dietary fatty acid intake and allergic response between men and women. It is very difficult from an epidemiological study to decide why that is. Obviously women have a different food intake pattern, less energy and less fat intake, and they also tend to be more conscious about the food selection in general. They may be better in reporting dietary patterns than many men. So it is very difficult to understand to which extent this is a biological difference, and to which extent it is a behavioral or other difference.

Dr. German: Does this polymorphism predict sensitivity to n-3 supplementation for anti-inflammatory effects?

Dr. Koletzko: It needs to be studied and perhaps with Dr. Prescott's data one could actually make a first step towards that. 
Dr. Nassar: Based on the anticipated benefits and despite the hazards, do you recommend supplementation of n-3 to patients with protein energy malnutrition?

Dr. Koletzko: Obviously n-3 fatty acids are essential substrates which one wants to include in a dietary supply. We tend to think that, at least in children, DHA is an essential substrate based on the observation that in children with PKU and a restricted intake of DHA, functional disturbances can be overcome with DHA supplementation. Probably children generally should have some supply of DHA. However, in a situation of nutritional rehabilitation after malnutrition, I would suggest that this should be closely studied. There are potential benefits similar to the situation in the critically ill. But once it has been studied, I think one would feel much more comfortable because one could not totally exclude that there might also be adverse effects. Obviously in a state of protein energy malnutrition there is often a reduction in anti-oxidative protection and it is conceivable that there might be more oxidative stress if omega 3 fatty acids are supplied, particularly in a combination with ascorbic acid, iron or other prooxidants. The other question regards a situation with a high risk of infection; if a high dose of an immunosuppressant substrate is given, there is a potential that infectious risk increases. I am not sure this would happen, I am not saying one should not do it, I am just saying in my view it would be worthwhile to study in a controlled fashion: supplement one group and another group not, and see what the effects are on such end points.

\section{References}

1 Koletzko B, Sauerwald T, Demmelmair H, et al: Dietary long-chain polyunsaturated fatty acid supplementation in infants with phenylketonuria: a randomized controlled trial. J Inherit Metab Dis 2007;30:326-332.

$\checkmark 2$ Agostoni C, Harvie A, McCulloch DL, et al: A randomized trial of long-chain polyunsaturated fatty acid supplementation in infants with phenylketonuria. Dev Med Child Neurol 2006;48: 207-212.

3 Beblo S, Reinhardt H, Demmelmair H, et al: Effect of fish oil supplementation on fatty acid status, coordination, and fine motor skills in children with phenylketonuria. J Pediatr 2007;150:479-484.

4 Koletzko B, Cetin I, Brenna JT, et al: Dietary fat intakes for pregnant and lactating women. Br J Nutr 2007;98:873-877.

5 Trak-Fellermeier MA, Brasche S, Winkler G, et al: Food and fatty acid intake and atopic disease in adults. Eur Respir J 2004;23:575-582. 
